\title{
An Efficient Analytical Technique for Fixed-Head Hydrothermal Scheduling
}

\author{
V. Vasudevan \\ Assistant Professor \\ Department of Electrical Engineering, \\ Annamalai University. Annamalainagar - 608002. \\ Tamilnadu, India.
}

\author{
P. Aravindhababu \\ Professor \\ Department of Electrical Engineering, \\ Annamalai University. Annamalainagar - 608002. \\ Tamilnadu, India.
}

\begin{abstract}
The short-term hydrothermal scheduling is a daily planning proposition in power system operation, a task which is usually more complex than the scheduling of all-thermal generation system. The classical iteration method involves time consuming three iterative loops for obtaining the optimal solution. This paper presents an analytical method for eliminating the two iterative loops of the classical iteration method with a view of enhancing the computational efficiency. It includes the simulation results of four test cases with a view to highlight its computational efficiency, irrespective of the problem size.
\end{abstract}

\section{Keywords}

Hydrothermal Scheduling, SHTS

\section{INTRODUCTION}

One of the most significant issues in power systems is short range hydrothermal scheduling (SHTS) that determines the hourly scheduling of available hydro and thermal generating units over the planning horizon, usually one day or one week. Due to insignificant operating cost of hydro plants, the most common objective function in SHTS intends to minimize the total fuel cost of thermal units, while satisfying various hydraulic and electric system constraints such as generation limits, available water and the energy balance equivalence over a scheduling horizon. It is a large-scale, dynamic, nonlinear and complicated constrained optimization problem [1].

Over the years, numerous mathematical methods with various degrees of near-optimality, efficiency, ability to handle difficult constraints and heuristics are suggested in the literature for solving the SHTS problems, such as gradient search technique [1], $\lambda-\gamma$ iteration method, dynamic programming [1], Lagrange relaxation [2], decomposition and coordination method [3], mixed integer programming [4], Newton's method [5]. linear programming [6], network flow programming $[7,8]$, non-linear programming [9] etc. Dynamic programming among these approaches has been found to tackle the complex constraints directly but suffers from the curse of dimensionality. The other methods have necessitated simplifications in order to easily solve the original model, which may lead to sub-optimal solutions with a great loss of revenue. Generally, these classical methods can be efficiently applicable for the SHTS problems with differentiable fuel cost function and constraints.

Recently artificial intelligence based methods, simulated annealing approach [10], evolutionary programming [11], genetic algorithm [12], artificial immune system [13], tabu search [14], ant colony optimization [15], particle swarm optimization [16], differential evolution [17], quantuminspired evolutionary algorithm [18] and artificial bee colony [19] are suggested for SHTS. These methods are able to provide good solution and deal with complicated nonlinear constraints more simply and effectively. Moreover, these algorithms do not depend on the first and second differentials of the objective function. However, the above mentioned methods require a large amount of computation time especially for large-scale SHTS problems.

In the light of the fact that each of these methods has its own merits and drawbacks, there is always a need for developing newer methods with a view of obtaining the global best solution at minimum computational efforts. A simple mathematical technique for solving fixed-head SHTS problem is suggested in this paper. This method eliminates the $\lambda$ iterations through an analytical approach in the classical $\lambda-\gamma$ iteration method with a view of enhancing the computational speed. The proposed algorithm is tested on three SHTS problems and the results are presented.

\section{PROBLEM FORMULATION}

The main objective of SHTS problem is to determine the optimal schedule of both hydro and thermal plants of a power system in order to minimize the total system operating cost, represented by the fuel cost required for the system's thermal generation. It is intended to meet the forecasted load demand over the scheduling period, while satisfying various system and unit constraints. The SHTS problem is formulated as

Minimize $\Phi=\sum_{k=1}^{n I n t} \sum_{i=1}^{n t} t_{k} \cdot F_{i k}\left(P_{T i k}\right)$

subject to the power balance constraint

$$
\begin{aligned}
& \sum_{i=1}^{n t} P_{T i k}+\sum_{j=1}^{n h} P_{H j k}-P_{D k}-P_{L k}=0 ; \\
& k=1,2, \cdots, n I n t
\end{aligned}
$$

and to the water availability constraint

$$
\sum_{k=1}^{n I n t} t_{k} \cdot Y_{i k}\left(P_{H_{i k}}\right)=V_{i}^{a v l} ; \quad i=1,2, \cdots, n h
$$

with

$$
\begin{gathered}
P_{T_{i}}{ }^{\min } \leq P_{T_{i k}} \leq P_{T_{i}}{ }^{\max } \\
i=1,2, \cdots, n t
\end{gathered}
$$


$P_{H_{i}}{ }^{\min } \leq P_{H_{i k}} \leq P_{H_{i}}{ }^{\max } \quad ; \quad i=1,2, \cdots, n h$

where

$F_{i k}\left(P_{T i k}\right)=a_{i} \cdot P_{T i k}^{2}+b_{i} \cdot P_{T i k}+c_{i} \quad \$ / h$

(5)

$Y_{i k}\left(P_{H i k}\right)=d_{i} \cdot P_{H i k}^{2}+e_{i} \cdot P_{H i k}+f_{i} m^{3} / h$

(6)

$$
\begin{gathered}
P_{L k}=\sum_{i=1}^{n g} \sum_{j=1}^{n g} P_{G i k} B_{i j} P_{G j k} \\
P_{G}=\left\{P_{T 1}, P_{T 2}, \cdots, P_{T n t}, P_{H 1}, P_{H 2}, \cdots, P_{H n h}\right\}
\end{gathered}
$$

\subsection{Classical $\lambda-\gamma$ iteration method [1]}

The augmented Lagrangian function for the SHTS problem is written as

$$
\begin{gathered}
\Phi_{T}=\sum_{k=1}^{n I n t}\left[\begin{array}{l}
\sum_{i=1}^{n t} t_{k} F_{i k}\left(P_{T i k}\right)-\lambda_{k} \\
\left.\left(\sum_{i=1}^{n t} P_{T i k}+\sum_{j=1}^{n h} P_{H j k}-P_{D k}-P_{L k}\right)\right]
\end{array}\right]+ \\
\sum_{i=1}^{n h} \gamma_{i}\left[\sum_{k=1}^{n I n t} t_{k} Y_{i k}\left(P_{H_{i k}}\right)-V_{i}^{a v l}\right]
\end{gathered}
$$

The co-ordination equation from the above function can be obtained as

$$
\begin{gathered}
t_{k} \frac{\partial F_{i k}\left(P_{T i k}\right)}{\partial P_{T i k}}+\lambda_{k} \frac{\partial P_{L k}}{\partial P_{T i k}}=\lambda_{k} \\
\gamma_{i} t_{k} \frac{\partial Y_{i k}\left(P_{H i k}\right)}{\partial P_{H i k}}+\lambda_{k} \frac{\partial P_{L k}}{\partial P_{H i k}}=\lambda_{k}
\end{gathered}
$$

The above co-ordination equations along with constraint Eqs. 2, 3 and 4 are iteratively solved to obtain optimal SHTS.

\section{PROPOSED METHODOLOGY}

The solution process of the $\lambda-\gamma$ iteration method involves time consuming three iterative loops, in which the $\lambda$-iterations itself accounts for two iterative loops in each $\gamma$ -iteration. The computational speed can be enhanced, if $\lambda$ iterations are eliminated, thereby avoiding two iterative loops. An analytical non-iterative approach is developed instead of $\lambda$-iterations in the proposed approach.

The co-ordination equation of the conventional $\lambda-\gamma$ iteration method, neglecting the losses can be written as,

$$
\begin{gathered}
t_{k} \cdot \frac{\partial F_{i k}\left(P_{G i k}\right)}{\partial P_{G i k}}=t_{k}\left(2 . a_{i} P_{G i k}+b_{i}\right)=\lambda_{k} \\
; \quad i=1,2 . . n t \\
t_{k} \cdot \frac{\partial Y_{i k}\left(P_{G i k}\right)}{\partial P_{G i k}}=t_{k}\left(2 . \gamma_{i} d_{i} P_{G i k}+\gamma_{i} e_{i}\right)=\lambda_{k} \\
; \quad i=n t+1, n t+2 . . n g
\end{gathered}
$$

Let the fuel cost and hydel discharge coefficient are redefined for the available (known) values of $\gamma$ as,

$$
\left.\begin{array}{l}
\left.\begin{array}{l}
a_{i}{ }^{\prime}=a_{i} \\
b_{i}{ }^{\prime}=b_{i} \\
c_{i}{ }^{\prime}=c
\end{array}\right\} \quad \text { for all thermal plants } i=1,2, \cdots, n t \\
a_{i}{ }^{\prime}=\gamma_{i} d_{i} \\
b_{i}{ }^{\prime}=\gamma_{i} e_{i} \\
c_{i}{ }^{\prime}=\gamma_{i} f_{i}
\end{array}\right\} \text { for all hydel plants } i=n t+1, n t+2, \cdots, n g
$$

Eqs. 12 and 13 can be written using the newly defined coefficients of Eqs. 14 and 15 as

$$
t_{k}\left(2 a_{i}{ }^{\prime} P_{G i k}+b_{i}{ }^{\prime}\right)=\lambda_{k} \quad ; \quad i \in \mathfrak{R}
$$

Where $\mathfrak{R}$ is a set of generators, initially it contains all the generators as

$$
\mathfrak{R}=\{1,2, \cdots, n t, n t+1, n t+2, \cdots, n g\}
$$

Rearranging Eq. (16) for optimal generation at interval-k as

$$
P_{G i k}=\frac{\lambda_{k}-b_{i}{ }^{\prime}}{2 a_{i}{ }^{\prime}} \quad ; \quad i \in \mathfrak{R}
$$

The above equation can be written in terms of $P_{D k}^{\Re}$ as

$$
P_{G i k}=\frac{P_{D k}^{\Re}-\rho-b_{i}{ }^{\prime} \sigma}{2 a_{i}{ }^{\prime} \sigma} ; \quad i \in \mathfrak{R}
$$

Where

$$
\rho=\sum_{i \in \Re} \frac{b_{i}{ }^{\prime}}{2 a_{i}{ }^{\prime}} \quad ; \quad \sigma=\sum_{i \in \mathfrak{R}} \frac{1}{2 a_{i}{ }^{\prime}}
$$

$P_{D k}^{\Re}$ is the power demand to be supplied by all the generators in the set of $\mathfrak{R}$. Initially it equals $P_{D k}$.

Eq. (19) provides optimal generations for the available values of $\gamma$ and minimizes the following cost function that involves the fuel cost of the thermal plants and the fictitious cost of the hydel plants.

$\operatorname{Min} \varphi_{k}=\sum_{i \in \Re} a_{i}{ }^{\prime} P_{G i k}{ }^{2}+b_{i}{ }^{\prime} P_{G i k}+c_{i}{ }^{\prime}$ 
Substituting Eq. (19) in Eq. (21) and rearranging

$$
\operatorname{Min} \varphi_{k}=A_{k} P_{D k}^{2}+B_{k} P_{D k}+C_{k}
$$

Where

$$
\left.\begin{array}{l}
A=\sum_{i \in \mathfrak{R}} \frac{1}{4 a_{i}{ }^{\prime} \sigma^{2}} \\
B=\sum_{i \in \mathfrak{R}} \frac{\rho}{2 a_{i}{ }^{\prime} \sigma^{2}} \\
C=\sum_{i \in \mathfrak{R}}\left(\frac{1}{4 a_{i}{ }^{\prime}}\right)\left(\frac{\rho^{2}}{\sigma^{2}}-b_{i}{ }^{2}\right)+C_{i}{ }^{\prime}
\end{array}\right\}
$$

Eq. (22) does not include the transmission loss. It can be included by altering the equation as

$$
\varphi_{k}=A_{k} P_{G k}^{\Re 2}+B_{k} P_{G k}^{\Re}+C_{k}
$$

where

$$
P_{G k}^{\Re}=\sum_{i \in \Re} P_{G i k}=P_{D k}^{\Re}+P_{L k}
$$

The $P_{L k}$ can be calculated by Eq.(7) through substituting the generations obtained by Eq.(19) for the set of generators in $\mathfrak{R}$ and the generations of limit violated generators.

Differentiating and equating Eq. (24) to zero yields the optimal $\lambda$ that minimizes $\varphi_{k}$

$$
\lambda^{o}=\frac{\partial \varphi_{k}}{\partial P_{G k}^{\Re}}=2 A_{k} P_{G k}^{\Re}+B_{k} \quad \$ / M W h
$$

The individual unit generation can be obtained by

$$
P_{\text {Gik }}=\frac{\lambda^{o}-b_{i}{ }^{\prime}}{2 a_{i}{ }^{\prime}} \quad \text { MW } ; \quad i \in \mathfrak{R}
$$

Though Eq. (27) provides the optimal generation $P_{G i k}$ at interval-k for the available $\gamma$ values, it may not satisfy the water availability constraint of Eq.(3). The SHTS problem may be solved iteratively for optimal $\gamma$ values that satisfy the water availability constraint. The algorithm is obtained below:

1. Read the system data

2. Choose initial $\gamma$-values for all hydel plants.

3. Set the interval $k=1$

4. $\quad$ Set $P_{D k}^{\Re}=P_{D k}$ and $\mathfrak{R}=\{1,2, \cdots, n t, n t+1, n t+2, \cdots, n g\}$

5. Evaluate the constants $\rho, \sigma, \mathrm{A}, \mathrm{B}$ and $\mathrm{C}$ for the generator in the set $\mathfrak{R}$
6. Compute the generation $P_{G i k}$ using Eq. (19) and then calculate $P_{L k}$ and $P_{G k}^{\Re}$ by Eqs. 7 and 25 respectively.

7. Evaluate $\lambda^{o}$ using Eq. (26) and then solve Eq. (27) for all the generators in the set $\mathfrak{R}$.

8. Check for limit violation of generators. If any of the generation violates, then set the respective limit as the generation by $P_{G i k}=P_{G i}^{\min }$ or $P_{G i}^{\max }$, eliminate the violated generator from the set $\mathfrak{R}$ reduce the power demand as $P_{D k}^{\Re}=P_{D k}^{\Re}-P_{G i k}$ and go to step (5).

9. Repeat steps 4-8 for all the intervals in the scheduling period.

10. Check for convergence through water availability constraints. If the algorithm converges, go to step 10 ; else, project new values for $\gamma$ and go to step 3

11. Optimal solution is obtained. Print the optimal generations and their cost

12. Stop

\section{NUMERICAL RESULTS}

The proposed method (PM) is tested on four SHTS problems, whose data comprising the cost characteristics of thermal plants, the discharge characteristics of hydel plants, their loss coefficients and water storage for each hydel plant are available in [20]. The first system under study comprises of one thermal and one hydel plant, the second unit has one thermal and two hydel plants, the third is made up of two thermal and two hydel plants and the last one contains of one thermal and one hydel plant. As the PM is an enhanced version of the classical $\lambda-\gamma$ iteration method, the results are compared with that of classical $\lambda-\gamma$ iteration method in order to exhibit the computational efficiency of the developed algorithm. The fuel costs obtained by the PM for all the test problems are presented in Table 1, which also includes the fuel costs of the $\lambda-\gamma$ iteration method. It is very clear from the results that the PM gives the same result of the $\lambda-\gamma$ iteration method, thereby indicating that the $\mathrm{PM}$ is as reliable as $\lambda-\gamma$ iteration method. The normalized execution time (NET) of the PM is compared with that of the $\lambda-\gamma$ iteration method for all the test problems in Table 2 . This table clearly indicates that the PM is much faster than that of the $\lambda-\gamma$ iteration method, thereby illustrating that the PM is computationally efficient. The optimal generations obtained by the PM for all the test problems are graphically presented in Fig.1. 


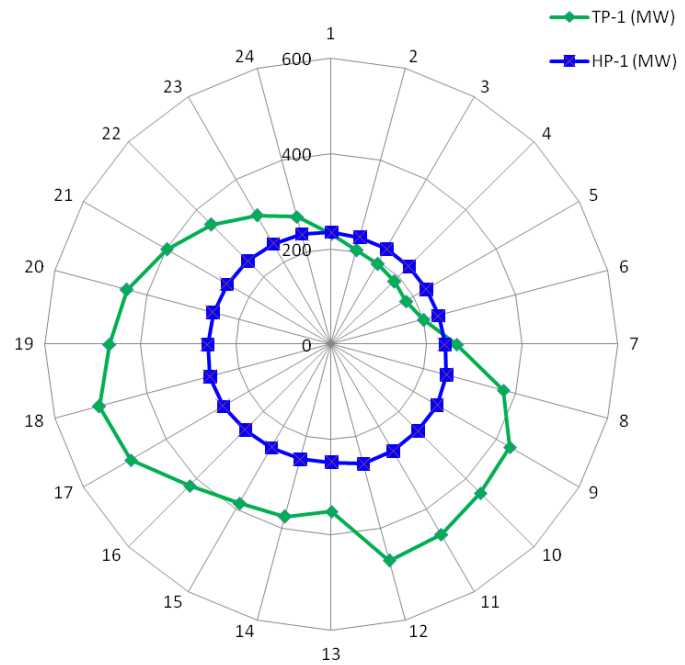

(a) Test Problem-1

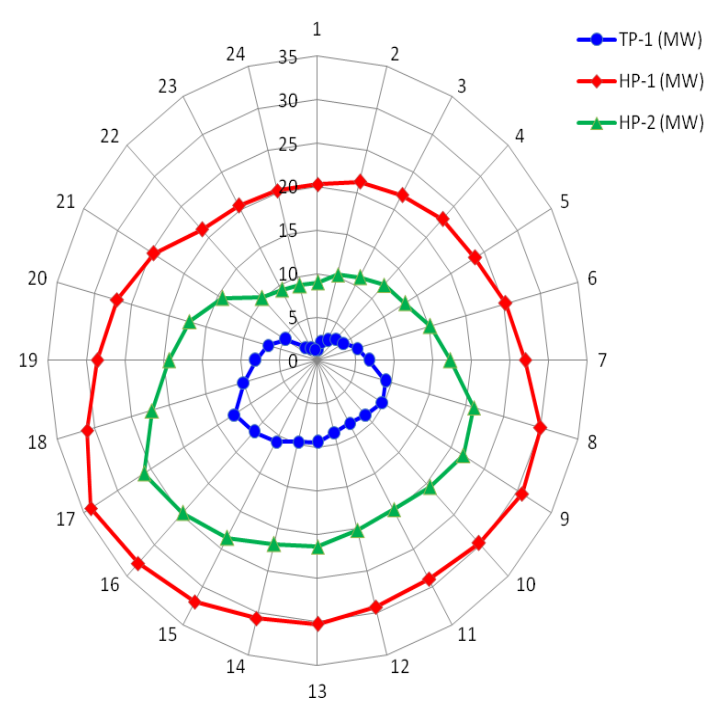

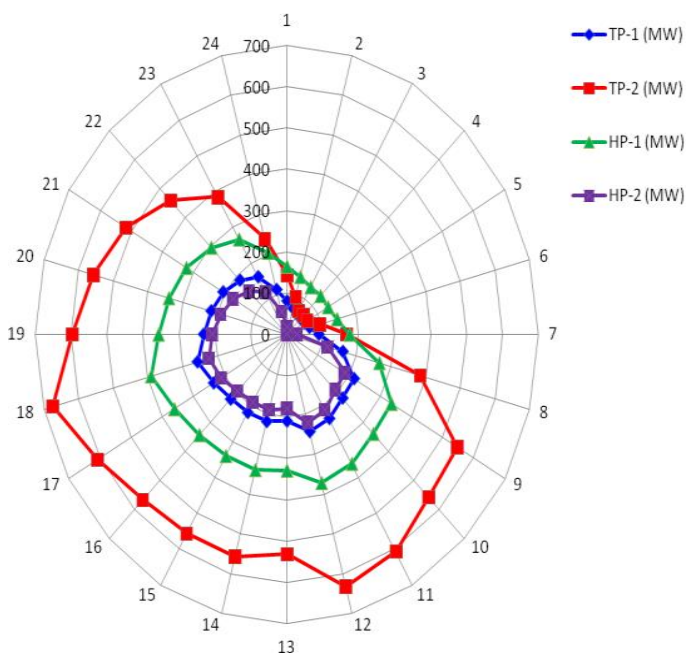

(c) Test Problem-3

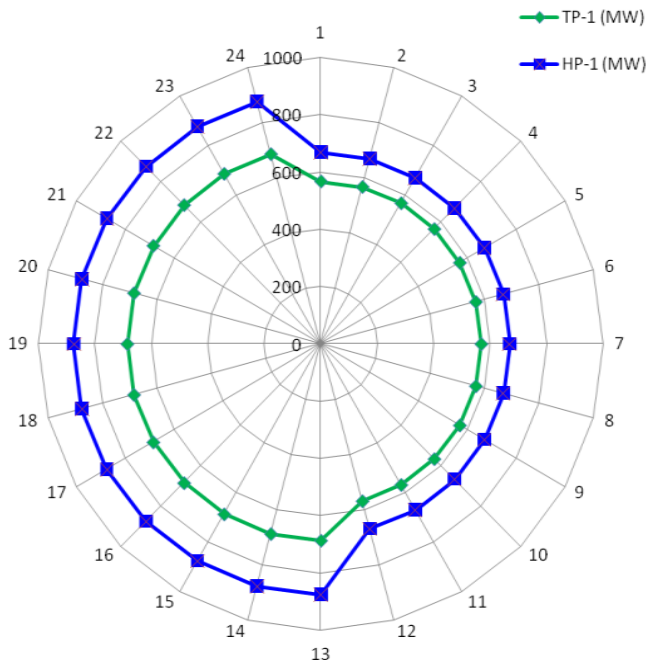

(d) Test Problem-4

(b) Test Problem-2

Fig.1 Optimal Generations obtained by the PM

Table-1 Comparison of Fuel cost

\begin{tabular}{|c|c|c|}
\hline Test Problem & $\lambda-\gamma$ iteration method & PM \\
\hline 1 & 96024.413 & 96024.413 \\
\hline 2 & 848.359 & 848.359 \\
\hline 3 & 53053.791 & 53053.791 \\
\hline 4 & 169637.597 & 169637.597 \\
\hline
\end{tabular}

Table-2 Comparison of NET

\begin{tabular}{|c|c|c|}
\hline Test Problem & $\begin{array}{c}\lambda-\gamma \text { iteration method } \\
(\mathrm{s})\end{array}$ & $\mathrm{PM}(\mathrm{s})$ \\
\hline 1 & 0.68 & 0.36 \\
\hline 2 & 0.87 & 0.54 \\
\hline 3 & 0.92 & 0.59 \\
\hline 4 & 0.62 & 0.33 \\
\hline
\end{tabular}




\section{CONCLUSION}

SHTS is one of the most important issues in the economic operation of power system. The objective of SHTS is to determine the optimal amount of water discharges of hydro plants and power generations of thermal plants over a scheduling horizon so as to minimize the total fuel cost of thermal plants while satisfying various hydraulic and electric system constraints. The classical $\lambda-\gamma$ iteration method involves time consuming three iterative loops for obtaining the optimal solution. An analytical method for eliminating the two iterative loops of the classical $\lambda-\gamma$ iteration method for SHTS with a view of enhancing the computational efficiency has been presented. The simulation results of four test cases clearly illustrated its computational efficiency.

\section{ACKNOWLEDGEMENTS}

The authors gratefully acknowledge the authorities of Annamalai University for the facilities offered to carry out this work.

\section{REFERENCES}

[1] Wood. AJ \& Woolenberg. BF. (1996). Power generation, operation and control, John Willey \& Sons, New York.

[2] Salam Nor KM, Hamdan AR.( 1998). Hydrothermal scheduling based Lagrangian relaxation approach to hydrothermal coordination. IEEE Trans Power Syst, 13(1):226-35.

[3] Li C, Svoboda AJ, Tseng Chung-Li, Johnson RB.( 1997). Hydro unit commitment in hydro-thermal optimization. IEEE Trans Power Syst, 12(2):764-9.

[4] Nilsson O, Sjelvgren D. (1996). Mixed-integer programming applied to short-term planning of a hydrothermal system. IEEE Trans Power Systems, 11(1): 2816.

[5] Zaghlool MF, Trutt FC. (1988). Efficient methods for optimal scheduling of fixed head hydrothermal power systems. IEEE Trans Power Syst, 3(1):24-30.

[6] Piekutowski M, Litwinowicz T, Frowd R. (1994). Optimal short-term scheduling for a large-scale cascaded hydro system. IEEE Trans Power Syst, 9(2):805-11.

[7] Li CA, Jap PJ, Streiffert DL. (1993). Implementation of network flow programming to the hydrothermal coordination in an energy management system. IEEE Trans Power Syst, 8(3):1045-53.
[8] Oliveira GG, Soares S. (1995). A second order network flow algorithm for hydrothermal scheduling. IEEE Trans Power Syst, 10(3):1635-41.

[9] Sjelvgren D, Brännlund H, Dillon TS. (1989). Largescale non-linear programming applied to operations planning. Int J Electr Power Energy Syst, 11(3): 213-7.

[10] Wong KP, Wong YW. (1994). Short-term hydrothermal scheduling, part-I: simulated annealing approach. IEE Proc Part-C, 141(5):497-501.

[11] Turkay B, Mecitog lu F, Baran S. (2011). Application of a fast evolutionary algorithm to short-term hydro-thermal generation scheduling. Energy Sourc, Part B: Econo, Plan, Policy:395-405.

[12] Sasikala J, Ramaswamy M. (2010). Optimal gamma based fixed head hydrothermal scheduling using genetic algorithm. Expert Syst Appl, 37(4):3352-7.

[13] Basu M. (2011). Artificial immune system for fixed head hydrothermal power system Energy, 36(1):606-12.

[14] Bai X, Shahidehpour SM. (1996). Hydro-thermal scheduling by tabu search and decomposition method. IEEE Trans Power Syst, 11(2):968-74.

[15] Huang SJ. (2001). Enhancement of hydroelectric generation scheduling using ant colony system based optimization approaches. IEEE Trans Energy Convers, 16(3):296-301.

[16] Wang Y, Zhou J, Zhou C, Wang Y, Qin H, Lu Y. (2012). An improved self-adaptive PSO technique for shorttermhydrothermal scheduling. Expert Syst Appl, 39(3): 2288-95.

[17] Basu M. (2014). Improved differential evolution for short-term hydrothermal scheduling. Int J Electr Power Energy Syst, 58:91-100.

[18] Wang Y, Zhou J, Mo L, Zhang R, Zhang Y. (2012). Short-term hydrothermal generation scheduling using differential real-coded quantum-inspired evolutionary algorithm. Energy, 44(1):657-71.

[19] Zhou J, Liao X, Ouyang S, Zhang R, Zhang Y. (2014). Multi-objective artificial bee colony algorithm for shortterm scheduling of hydrothermal system. Int J Electr Power Energy Syst, 55:542-53.

[20] Abdul Halim, Abdul Rashid and Khalid Mohamed Nor. (1991). An efficient method for optimal scheduling of fixed head hydro and thermal plants, IEEE Trans. on Power Systems, 6(2), 631-636. 